\title{
PARYLENE NEURO-CAGES FOR LIVE NEURAL NETWORKS STUDY
}

\author{
Qing He, Ellis Meng, Yu-Chong Tai, Christopher M. Rutherglen*, Jon Erickson*, and Jerome Pine* \\ Department of Electrical Engineering and Department of Physics* \\ California Institute of Technology, CA 91125, USA
}

Tel.: (626) 395-3884, Fax: (626) 584-9104, email: qing@mems.caltech.edu

\begin{abstract}
Here we present a surface-micromachined Parylene neuro-cage array for the in vitro study of live neural networks. Various types of neuro-cages have been fabricated and several adhesion promotion techniques have been explored. The biocompatibility and mechanical robustness of Parylene neuro-cages have been demonstrated through cell culture experiments. Dissociated neuronal cell bodies have been inserted one to a cage and cultured, allowing neurites to grow out through Parylene channels and form live neural networks. In addition, there is no observable difference between neural growth on Parylene and on oxide surfaces. It is possible to use the same or slightly modified cage structures to accommodate different cells, leading to possible applications of this technology in cell study and drug discovery.
\end{abstract}

\section{INTRODUCTION}

Studying how neural networks, formed by individual neurons, perform in detail is the central task of neuroscience. Live neural networks have been extensively researched. The conventional technique is to use planar arrays of extracellular metal electrodes on which neural cultures are grown [1-2]. However, this approach can only arbitrarily select and access a small proportion of neurons in the entire network. Furthermore, since neurons are mobile, repeated measurements of a specific neuron are difficult to obtain, especially for long-term experiments.

In order to study each neuron in the neural network, one-to-one correspondence between the neurons and electrodes has to be established and maintained. This can be achieved by physically confining individual neurons over corresponding electrodes without affecting neurite growth and neural network formation. To accomplish these goals, our approach is to use micro-cage, like a micro Skinner Box, trapping each neuron into one cage while still allowing neurites to grow out through long thin channels connected to the cages. The neuro-cages are constructed in arrays to allow neurites from different neurons to form neural networks. Thus the cultured neurons in a network can be reliably stimulated and monitored individually and continuously over long periods of time.

\section{DESIGN}

Previous work from this lab developed open-faced neuro-wells for cell containment and measurement
(Figure 1), which has successful achieved the stated goals [3-4]. However, several drawbacks were identified. First, the process of making bulk micromachined well"with nitride canopy and channels is very complicated and had low yield. In addition, neurites growing out through channels on top of the well tended to pull neuron away from well-bottom electrodes. To address these issues, a more suitable technology using Parylene and thick photoresist was developed incorporating a new structural design consisting long thin channels radiating from the base of the cage.

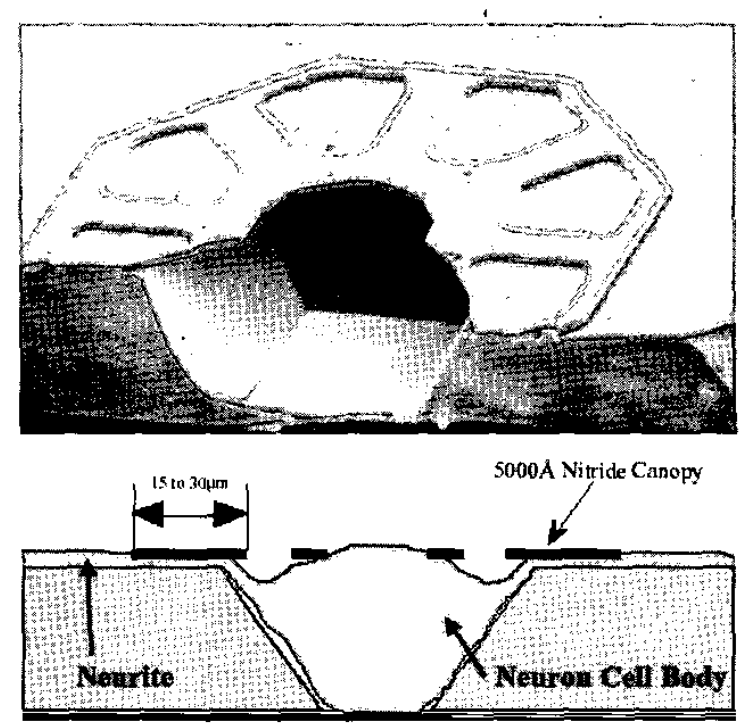

Figure 1. Neuro-well previously developed in our group.

The structure of the new surface-micromachined neuro-cages is illustrated in Figure 2. Parylene is chosen as the structural material in this application for its unique properties. It is non-toxic, extremely inert, resistant to moisture and most chemicals, and biocompatible. These properties make Parylene well suited for long-term cell culture experiments. Its conformal deposition makes it easy to fabricate $3 \mathrm{D}$ structures like the neuro-cage, thus simplifying the fabrication process as compared to the bulk micromachined neuro-well. Also, yield is no longer a problem. Low-temperature deposition makes CMOS integration possible. Most importantly, Parylene is transparent. Thus neurons can be seen under microscope through the Parylene cages and neurites are easily observed as they grow through the channels. Because neurons can grow on oxide surfaces, the Parylene cages are attached to an oxidized silicon substrate. 


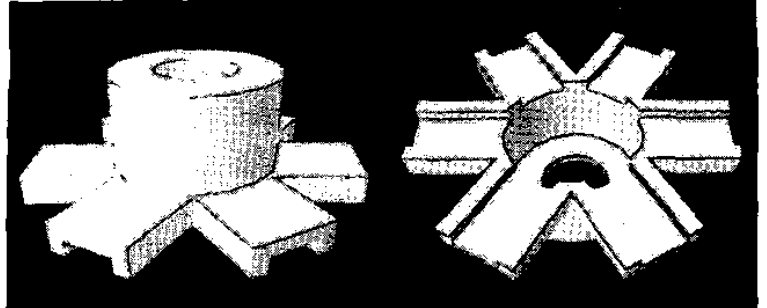

Figure 2. 3D illustration of the neuro-cage side and bottom view.

The neuro-cages are arranged in a 4 by 4 array for studying a small 16-neuron neural network. The microcages, spaced $100 \mu \mathrm{m}$ apart, are designed to hold a single neuron in each cage. The cage consists of a top loading access hole, the cage body, and 6 thin channels that protrude from the bottom of the cage. Rat embryonic hippocampal neurons are used in our study. These neurons are 12 to $15 \mu \mathrm{m}$ in diameter when freshly dissociated, and when matured, are about $20 \mu \mathrm{m}$ in diameter and $10 \mu \mathrm{m}$ in thickness. Given these dimensions, the neuro-cages are designed to have a diameter of $30 \mu \mathrm{m}$, height of $15 \mu \mathrm{m}$, and access hole diameter of $15 \mu \mathrm{m}$ (Figure 4). Currently, the Parylene neuro-cages are used in culture viability experiments and no electrodes are incorporated.

To guarantee that a specific neuron can be repeatedly measured and stimulated, the neuron has to be kept in close proximity to an electrode. However, it is well known that neurons tend to move. In our previous research, it was observed that neurons could manage to escape from even $1 \mu \mathrm{m}$ by $3 \mu \mathrm{m}$ holes at the cost of cell damage or death. Therefore, long thin channels are designed as neurite outgrowth path to prevent neurons from escaping. This design has been shown to work well in cell culture experiments.

\section{FABRICATION}

Various neuro-cages with different dimensions are fabricated using similar processes. The generalized fabrication flow is shown in Figure 3. First an oxide layer is grown on silicon wafer. A channel height controlling sacrificial layer is then patterned. Two Parylene layers and one photoresist layer are used to form the cage. AZ9260 photoresist is used to make the $15 \mu \mathrm{m}$-thick cage body and nearly vertical sidewalls are achieved (Figure 4). The sacrificial materials are finally removed to release the microcage.

Experimentally, it has been shown that channel height is critical in achieving both neurite growth and neuron trapping. Therefore this process is designed to make the channel height adjustable over a wide range, by using different sacrificial materials. For thin channels approximately $0.5 \mu \mathrm{m}$ thick (Figure $5(\mathrm{a})$ ), sputtered silicon was used as the sacrificial material and later removed by $\mathrm{BrF}_{3}$ or $\mathrm{XeF}_{2}$ gas etching. Gas phase etching is advantageous in avoiding stiction which is normally experienced when releasing thin channels using wet etchant.

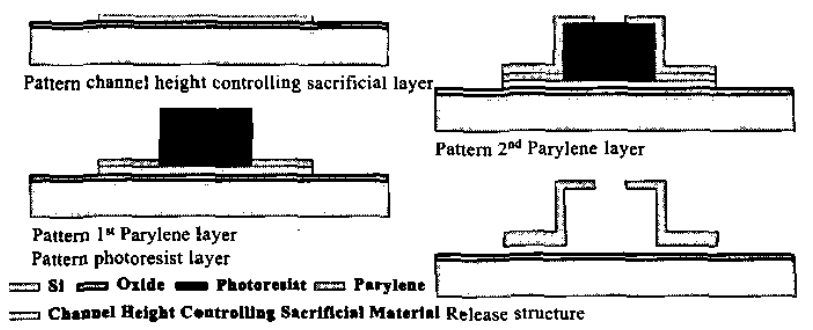

Figure 3. Generalized fabrication flow of the neuro-cages.

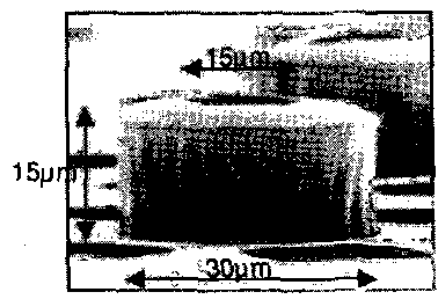

Figure 4. SEM picture of a fabricated neuro-cage.

For even thicker channels $(\sim 2 \mu \mathrm{m})$ (Figure 5(b)), hardbaked photoresist is used as the sacrificial material. The photoresist AZ1518 was hardbaked at $140 \mathrm{C}$ for $30 \mathrm{~min}$ and later removed in ST-22 photoresist stripper. The hardbake serves two purposes. One is to allow the channel photoresist survive patterning of the sacrificial photoresist used to form neuro-cage body. The other is to withstand the isopropyl alcohol used in A-174 (gammaMethacryloxypropyltrimethoxy Silane) Parylene adhesion promotion solution (A-174: DI Water: IPA=1:100:100).

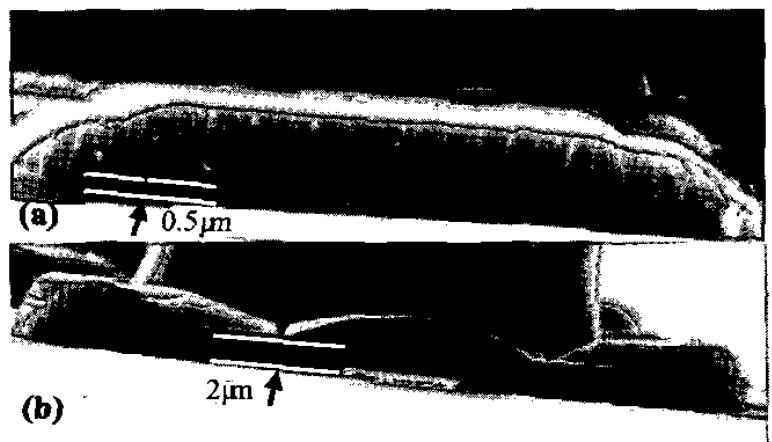

Figure 5. Neuro-cage channels with different channel heights. (a) $0.5 \mu \mathrm{m}$-high channel with $3 \mu \mathrm{m}$ Parylene; (b) $2 \mu \mathrm{m}$-high channel with 2 m Parylene.

Efforts have also been made to improve the adhesion of the neuro-cages to the substrate. While Parylene-tooxide adhesion is usually improved by applying A-174 to the substrate before Parylene deposition, this is insufficient for withstanding immersion in aggressive 
chemicals used in sterilization and cell culture solutions for long periods of time. This is partly due to the small Parylene-to-oxide contact area. The chemical adhesion layer in this region is easily compromised. Therefore, two alternative robust adhesion promotion techniques are investigated. The first method relies on mechanically anchoring Parylene to a silicon substrate [5]. DRIE is used to etch a trench with a mushroom-like cross section. The bottom of the trench is wider and serves to anchor deposited Parylene layers. The other technique is to roughen the anchoring area with short time etching in $\mathrm{BrF}_{3}$ or $\mathrm{XeF}_{2}$. Both methods dramatically increase the contact area for adhesion.

The filled-trench process (Figure 6) starts with patterning oxide layer on silicon wafer to expose silicon in the trench/anchor areas. Then $0.3 \mu \mathrm{m}$ silicon and $0.2 \mu \mathrm{m}$ $\mathrm{Al}$ are sputtered. First, $\mathrm{Al}$ is patterned and chemically etched. Then sputtered silicon is patterned and etched in DRIE using photoresist and $\mathrm{Al}$ as mask. $15 \mu \mathrm{m}$-thick photoresist AZ9260 is defined to form the cage body. Nearly vertical sidewalls are achieved (Figure 7(b)). DRIE is then used to make the anchoring structures using a modified Bosch process. The standard procedure is used to etch a $10 \mu \mathrm{m}$ deep trench. Then an isotropic etch in $\mathrm{SF}_{6}$ plasma is performed to create a widened portion at the bottom of the trench. Parylene is deposited and patterned, followed by sacrificial materials removal. Figure 7 shows the fabricated neuro-cages and anchor cross sections.

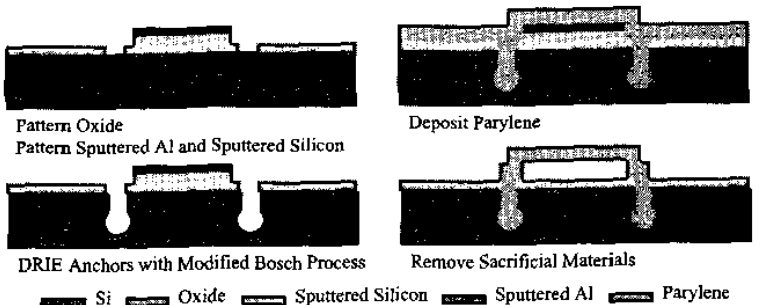

Si Oxide Sputtered Silicon $\longrightarrow$ Sputtered Al

Figure 6. Process flow of neuro-cages with Parylene-filled anchors. The figure shows cross section of cage channels.

The roughening processing also starts with patterning oxide to expose silicon in the anchor areas. A brief etch in $\mathrm{XeF}_{2}$ gas is then used to roughen the silicon surface. The following process is the same as the process in Figure 3. The illustration and fabricated cage is shown in Figure 8.

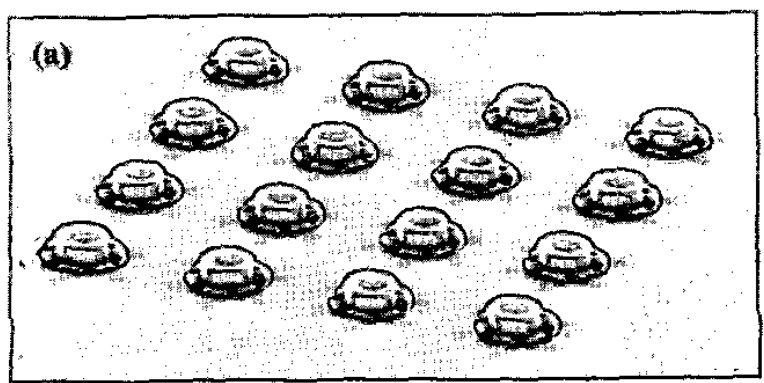

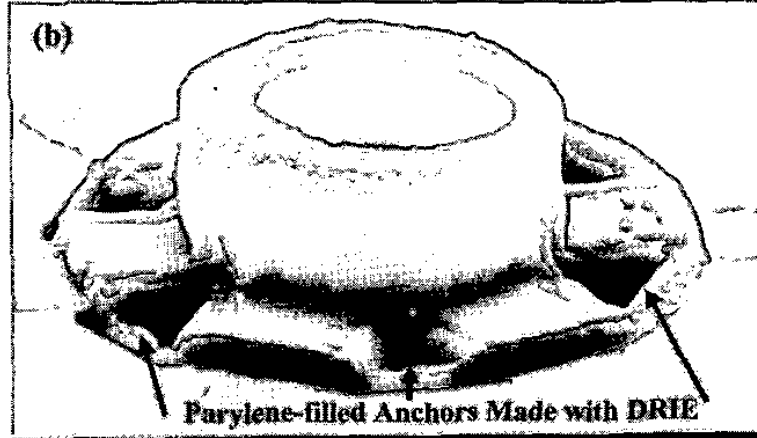

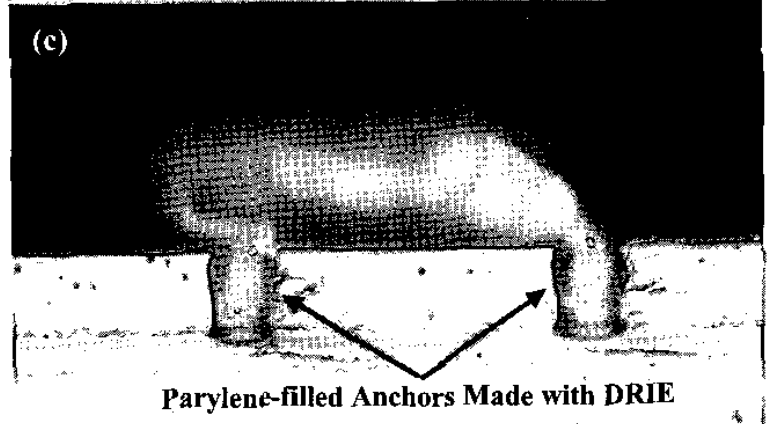

Figure 7. Fabricated neuro-cages with DRIE-made Parylene-filled anchors. (c) is the cross section of anchors.

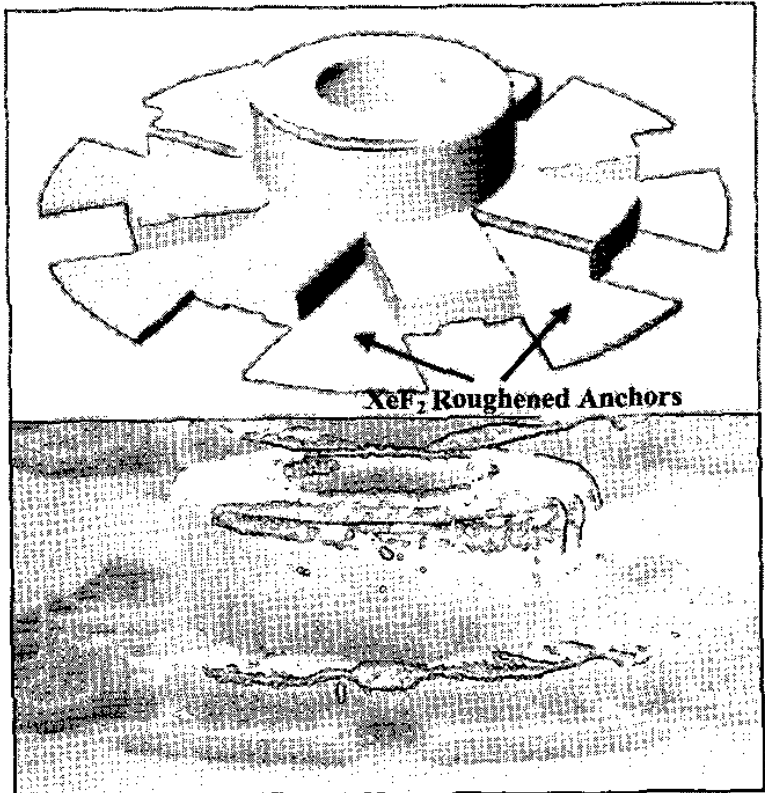

Figure 8. Illustration and fabricated neuro-cages using $\mathrm{XeF}_{2}$ roughened anchors.

\section{CELL CULTURE EXPERIMENTS}

The fabricated chips are first sterilized to prevent bacteria from infecting neural cultures. Rat hippocampal neurons are loaded into the cages through the loading hole by manually sucking neurons up in a glass pipette and injecting them into neuro-cages one-by-one. Since 
Parylene is transparent, the neurons can be seen under microscope when they are placed into the neuro-cages. Neurons are then cultured and observed for developments. Usually neurons grow neurites that spread out to form neural networks after several days in culture.

First, Parylene has been shown to be compatible with neurons and glial cells. Figure 9 shows the growth of neurons and glial cells on a Parylene surface. There is no observable difference between neural and glial growth on Parylene and oxide surfaces. Neurons are observed to grow multiple processes that are long and branching, which is an indication of healthy cells. Also, neural networks were formed.

Secondly, the Parylene neuro-cages are shown to be functional as designed. As shown in Figure 10, freshly dissociated neuron can be loaded into neuro-cage. Neurites have successfully grown out from cage channels, while the neuron cell body is still trapped inside the cage.

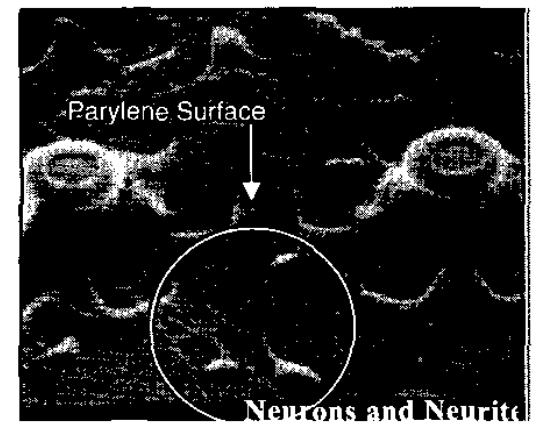

Figure 9. Neural and glial growth on Parylene surface.

\section{CONCLUSION}

Various types of surface-micromachined Parylene neuro-cages have been fabricated to study live neural networks. Through cell culture experiments using dissociated rat hippocampal neurons, the Parylene neurocages have been verified to be both biologically compatible and mechanically functional.

\section{ACKNOWLEDGEMENTS}

This work is supported by the NSF ERC program at Caltech (Award No. EEC-9402726) and NIH (Award No. R01 NSO44134).

\section{REFERENCES}

[1] J. Pine, Recording action potentials from cultured neurons with extracellular microcircuit electrodes", Journal of Neuroscience Methods, 2, 1980, 19-31.

[2] Y. Jimbo, T. Tateno, and H.P.C. Robinson, "Simultaneous Induction of Pathway-Specific Potentiation and Depression in Networks of Cortical Neurons"' B iophysical J., Vol. 76, Feb. 1999, 670-678.
[3] J.A. Wright, S.T. Lucic, Y.-C. Tai, M.P. Maher, H. Dvorak and J. Pine, Towards a functional MEMS neurowell by physiological experimentation,' ASME Int. Mechanical Engineering Congress and Exposition, DSCVol. 59, Atlanta, GA, pp. 333-338, Nov. 1996.

[4] M.P. Maher, J. Pine, J. Wright and Y.-C. Tai, "The neurochip: a new multielectrode device for stimulating and recording from cultured neurons"' Journal of Neuroscience Methods, 87, 1999, 45-56.

[5] M. Liger, D.C. Rodger, and Y.-C. Tai, Robust Parylene-to-Silicon Mechanical Anchoring," $16^{\text {th }}$ IEEE Int. Conf. on Micro Electro Mechanical Systems (MEMS 03), Kyoto, Japan, Jan. 2003, pp. $602-605$.
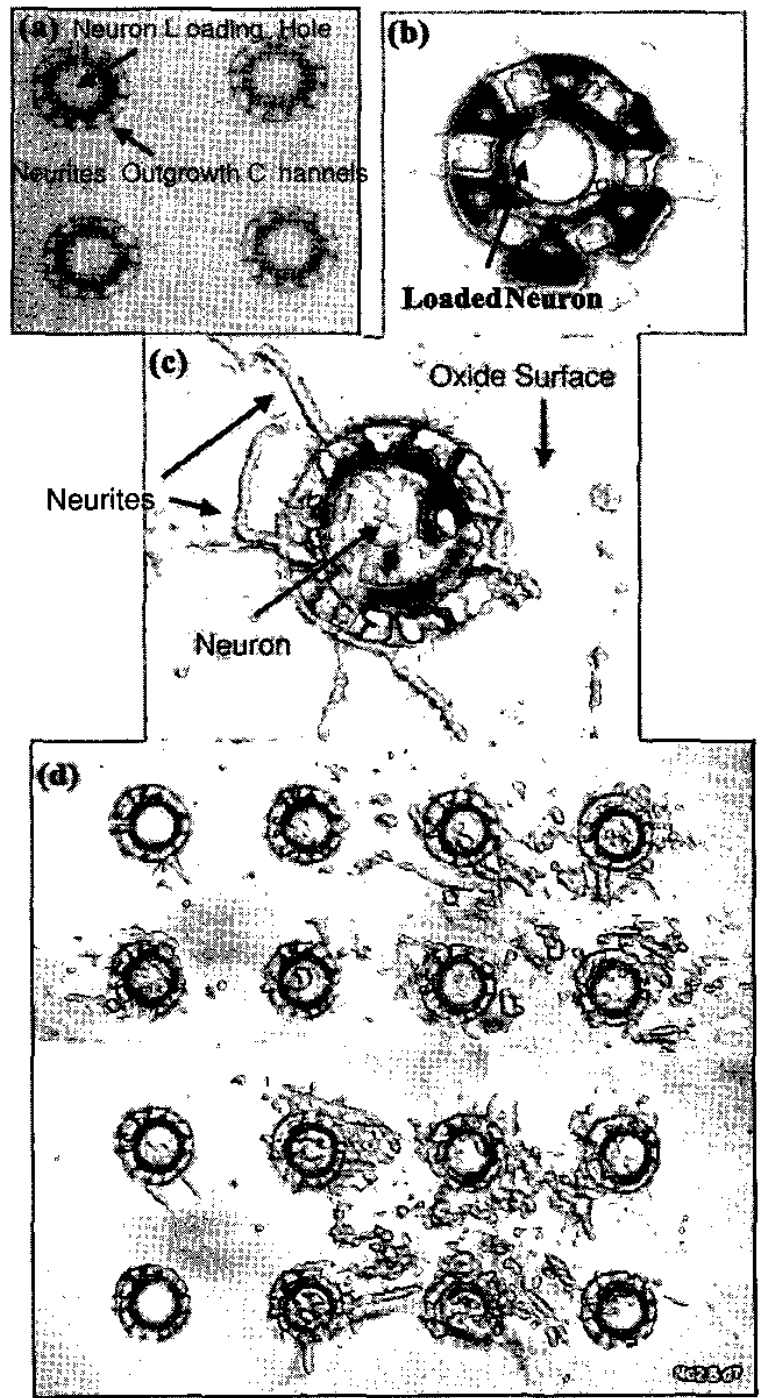

Figure 10. (a) Optical picture of neuro-cages ready to be loaded; (b) A neuron just loaded into a neuro-cage; (c) Nomarski picture of neurites outgrowth through cage channels while neuron cell body is trapped inside the cage; (d) Mosaic Nomarski picture of neural and glial growth in a 4 by 4 neuro-cage array after seven days in culture. Note not all cages were loaded with neuron. 